\title{
José Carlos Santos Paz (dir.), La obra de Gebenon de
} Eberbach

Florence : SISMEL, Edizioni del Galluzzo, 2004, CCCXLII+142 p., $74 €$.

\section{Laurence Moulinier-Brogi}

\section{OpenEdition}

\section{Journals}

Édition électronique

URL : http://journals.openedition.org/ifha/8398

DOI : 10.4000/ifha.8398

ISSN : 2198-8943

Éditeur

IFRA - Institut franco-allemand (sciences historiques et sociales)

Référence électronique

Laurence Moulinier-Brogi, « José Carlos Santos Paz (dir.), La obra de Gebenon de Eberbach », Revue de I'IFHA [En ligne], Date de recension, mis en ligne le 01 février 2016, consulté le 22 septembre 2020. URL : http://journals.openedition.org/ifha/8398 ; DOI : https://doi.org/10.4000/ifha.8398

Ce document a été généré automatiquement le 22 septembre 2020

(CIFHA 


\section{José Carlos Santos Paz (dir.), La obra de Gebenon de Eberbach}

Florence : SISMEL, Edizioni del Galluzzo, 2004, CCCXLII+142 p., $74 €$.

Laurence Moulinier-Brogi

En canonisant en octobre 2012 la bénédictine rhénane Hildegarde de Bingen (1098-1179), Benoit XVI a fait mentir Kurt Flasch, qui écrivait avec humour il n'y a pas si longtemps que «la sainte abbesse Hildegarde de Bingen n'était même pas originaire de Bingen et ne fut ni abbesse, ni sainte ! (K. Flasch, Philosophie hat Geschichte, Band 1, 2003, "Hände weg von Hildegard! », p. 342). De fait, si Hildegarde était passée à la postérité comme sainte prophétesse inspirée malgré trois tentatives avortées de canonisation, c'est surtout grâce à l'action d'un homme en particulier, Gebeno d'Eberbach, dont José Carlos Santos Paz propose ici la première édition critique de son Speculum futurorum temporum accompagnée d'une étude philologique des sources, et d'une reconstitution exemplaire de la tradition manuscrite, des problèmes textuels encore posés par le Speculum, mais aussi de la biographie de l'auteur et de l'influence de son œuvre.

On sait peu de chose de la vie de Gebeno, si ce n'est qu'il était sous-prieur en 1213 et prieur en 1215, de la toute jeune abbaye cistercienne d'Eberbach, fondée au bord du Rhin par l'archevêque de Mayence Adalbert Ier (1110-1111/1137), qui y avait installé dans un premier temps des chanoines réguliers avant de faire appel à des cisterciens de la filiation de Clairvaux. On ignore quand disparut Gebeno, qui était encore en vie en 1223. Ce que l'on sait en revanche, comme il le dit lui-même, c'est qu'il composa entre 1217 et 1220 , sous le titre de Speculum futurorum temporum, un montage des prédictions de Hildegarde pour faire pièce aux prophéties sur la venue de l'Antéchrist qui circulaient à son époque, notamment celles de Joachim de Flore (v. 1132-1202). Joachim avait été lui-même cistercien avant d'entrer en conflit avec l'ordre et en 1215, il avait été condamné pour certaines de ses opinions trinitaires lors du $4 \mathrm{e}$ concile de Latran. Et en 1217, Gebeno avait reçu la visite d'un abbé calabrais du nom de Juan apparemment déterminante pour son passage à l'écriture. 
Quant à Hildegarde, elle avait été en contact épistolaire tant avec la communauté d'Eberbach dans son ensemble qu'avec certains de ses membres : on conserve six lettres adressées par Hildegarde à Eberbach, et sa mémoire et ses œuvres y étaient encore fort présentes du temps de Gebeno, qui dans la seconde version de l'épitre dédicatoire dont il munit son ouvrage, que l'on peut dater de 1222 environ, se montre capable de donner une liste des écrits de l'abbesse.

Dans une introduction aussi riche qu'érudite de plus de trois cents pages, divisée en cinq chapitres (le dernier étant consacré aux principes de l'édition critique et à différents stemmas), José Carlos Santos Paz fait d'abord le point sur l'œuvre de Gebeno : n'est-il que l'homme d'une compilation ou a-t-il écrit d'autres choses? Il faut rappeler qu'on attribue à tort à Gebeno des écrits auxquels il est ici fait justice (p.XXII), et José Carlos Santos Paz répartit les écrits du prieur en deux groupes: des extraits, auxquels appartiennent tant le Speculum que ses écrits sur la vie religieuse en communauté, et les commentaires comme la lettre aux moniales du Rupertsberg, un petit traité sur l'Apocalypse (De hereticis ex Apocalypsi) ou encore une note sur le schisme, dont il fournit également une édition dans ce volume.

Une fois posée l'existence de ces deux rédactions, Jose Carlos Santos Paz en vient à formuler des hypothèses sur la composition de l'œuvre (p. LXXIV). Pour parvenir à ses fins, Gebeno puisa tant dans les livres que dans les lettres de Hildegarde, pour prouver que l'attente joachimite d'une prochaine arrivée de l'Antéchrist était une erreur. Avant cela, de nombreuses époques devaient encore passer, et il fallait les exploiter pour en revenir à la réforme.

Les sources de Gebeno autres que Hildegarde sont passées en revue, mais c'est évidemment sur l'organisation du matériau hildegardien par Gebeno que porte l'essentiel de l'analyse de José Carlos Santos Paz et sur l'identité des codices qui lui servirent. L'analyse textuelle qu'il a menée lui permet par exemple d'affirmer (p. XXXI) que ni le Riesenkodex, codex géant formant comme le canon des œuvres de la moniale copiée de son vivant au monastère qu'elle avait fondée, le Rupersberg, ni le Scivias de l'abbaye d'Eberbach ne furent ses vrais modèles: la comparaison patiente du texte de Gebeno avec différents témoins du Scivias lui permet d'avancer qu'aucun des codices connus à ce jour ne fut le véritable modèle du compilateur et que c'est le manuscrit de Troyes, ou un codex très similaire, qui reste le meilleur candidat (p. XXXVI).

José Carlos Santos Paz distingue deux états du Speculum, dont on connaît une première rédaction incomplète et provisoire par plusieurs manuscrits : la plus diffusée, la plus connue, fut la première, dont les manuscrits connus et ici inventoriés sont bien plus nombreux que ceux de la seconde, mais il relève aussi que les deux rédactions eurent des interférences, des contaminations, comme il ressort de l'analyse du ms. de Munich, par exemple.

Le quatrième chapitre de cette introduction est un précieux catalogue : José Carlos Santos Paz inventorie les 108 manuscrits conservés, liste les manuscrits perdus, et recense également les manuscrits "douteux ", c'est-à-dire ceux qui, sans renfermer véritablement l'œuvre de Gebeno, contiennent qui une Visio qui une Prophetia Hildegardis, titres sous lesquels la compilation du cistercien était également fréquemment désignée. Et il faut souligner ici que ce grand nombre de manuscrits contraste avec le faible nombre d'exemplaires des propres écrits de Hildegarde: sa première et célèbre œuvre visionnaire intitulée Scivias n'est transmise que dans onze 
manuscrits complets et l'on ne connaît actuellement que quatre témoins du Livre des cuvres divines et cinq du Livre des mérites de la vie.

De fait, cette compilation beaucoup plus diffusée que les propres écrits de Hildegarde assura à la nonne une célébrité durable bien au-delà du Moyen Âge, en même temps qu'elle en transmettait une image déformée et son entreprise suscita d'emblée des critiques : dès 1220, une sœur du Rupertsberg, le taxe de transgressor des écrits de sa sainte patronne (p. LXXV), se fondant sur les derniers mots, très comminatoires du Liber divinorum operum: "Qu'aucun homme ne soit donc assez téméraire pour faire quelque ajout aux termes de cet écrit ou pour en supprimer quelques passages s'il ne veut pas être éliminé du livre de vie et de toute la béatitude terrestre ", avertissait Hildegarde.

Gebeno déplorait qu'elle ne soit guère lue à cause de l'obscurité de son style (ce qui était, d'après lui, la marque des prophètes : ms. Paris, BnF, lat. 3322, In hoc autem libello non omnia posui que in libris eius de futuris scripta sunt sed ea que huic operi et isti tempori congruere putaui [...] Libros sancte Hildegardis plerique legere fastidiunt et horrent pro eo quod obscure et inusitato stilo loquitur, non intelligentes quod hoc est argumentum vere prophecie, fol. 4) ; il s'employa donc à rendre accessibles ses écrits en les réorganisant, et chercha aussi à lester les prophéties de Hildegarde du poids d'une autorité incontestable. Quitte à faire subir quelques entorses à la réalité des faits, il affirma que les livres de Hildegarde avaient été officiellement approuvés, non par des maitres de théologie mais par le pape en personne: Praeterea sciendum est quod libri sanctae Hildegardis recepti et canonizati sunt a papa Eugenio in concilio Trevirensi, praesentibus multis episcopis tam Francorum quam Teutonicorum, et sancto Bernardo abbate Clarevallensis.

Or en affirmant le premier que les livres de Hildegarde avaient été canonizati par Eugène III, Gebeno lança une expression qui fut très largement reprise par la suite et qui peut expliquer que Hildegarde ait bénéficié aux yeux de nombreux auteurs d'une canonisation obtenue en quelque sorte par métonymie.

Progressivement, les successeurs ou épigones de Gebeno soulignèrent que les livres de Hildegarde avaient avant tout prédit l'arrivée de nouveaux ordres, et c'est ainsi que la nonne se retrouva à la tête d'une prophétie dans laquelle elle aurait prédit la naissance des Ordres mendiants - et de ce fait mêlée au conflit qui oppose les ordres nouveaux à leurs adversaires, particulièrement violent entre 1255 et 1270 . Un des principaux acteurs de ce conflit, le maître séculier Guillaume de Saint-Amour, chargea les Franciscains en s'appuyant notamment sur Hildegarde, les accusant d'être de pseudoprophètes; il avait en fait vu à Clairvaux la compilation de Gebeno et il rappelle à son tour que les écrits de la nonne ont été «canonisés ». C'est peut-être lui aussi qui composa la prophétie Insurgent gentes, thématiquement très proche des vraies prophéties de Hildegarde et très inspirée par la lettre qu'elle adressa au clergé de Cologne vers 1163.

L'utilisation des prophéties de Hildegarde à diverses fins dans des contextes de division religieuse ou politique, voire de schisme ne s'arrête pas là, tant s'en faut : Pierre Jean Olivi l'utilise dans son commentaire à l'Apocalypse ; Jean de Roquetaillade sollicite ses textes, et insiste sur l'amitié supposée de Hildegarde et Bernard de Clairvaux afin de donner à ses propres écrits la caution d'une sainte authentique - et de ce point de vue, un des multiples apports du travail de José Carlos Santos Paz réside aussi dans une édition du dixième traité du Liber ostensor de Roquetaillade mettant clairement en lumière l'inspiration gebenonienne (p. CLV-CLVII). 
Henri de Langenstein († 1397), quant à lui, après une carrière de théologien à Paris, se retira pendant un an environ à l'abbaye d'Eberbach et se servit des prophéties de Hildegarde, faisant un vif éloge, à propos du Schisme, de la Theotonicorum Sibilla qui en avait, selon lui, prophétisé le déclenchement ; pendant la Réforme, même, on demanda à Hildegarde de cautionner telle ou telle doctrine, et au XVIII siècle, par l'intermédiaire des Annales ecclésiastiques de Bzovius relayé par le Dominicain Jeronimo Baptista de Lanuza, elle fournit encore la matière de satires dirigées contre les Jésuites !

Dès le début du XIII siècle, les visions de Hildegarde sorties de leur contexte par Gebeno et en quelque sorte nimbées d'atemporalité purent donc être employées au service d'objectifs très divers, et ce trait s'accentua en raison du climat dans lequel l'ordre franciscain prit son essor, très marqué par les prophéties de Joachim de Fiore. Mais c'est bien sûr la procédure d'extraits, à laquelle s'était livré Gebeno, qui permit à des contextes très différents d'y trouver de quoi appuyer leur combat, bien que Gebeno eût affirmé, au début du Pentachronon, n'avoir sélectionné que ce qui se rapportait à son époque (Igitur ad confutandos et convincendos huiusmodi pseudo prophetas libellum hunc compilavi; maxime autem et specialiter propter emendationem et correctionem claustralium et cleri eum descripsi [...] in hoc autem libello non omnia posui que in libris ejus de futuris scripta sunt, sed ea que huic operi et isti tempori congruere putavi).

La moisson de l'entreprise du prieur d'Eberbach s'avère donc triple : il a contribué à assurer le rayonnement de la prophétesse, beaucoup plus que les propres écrits de Hildegarde ; cette image qu'il a voulu imposer, quitte à la déformer, put se révéler à double tranchant; enfin, Gebeno a réussi à diffuser durablement la figure d'une prophétesse dont les écrits avaient été officiellement reconnus par un pape, canonizati, alors que la canonisation officielle de la religieuse n'eut jamais lieu au Moyen Âge.

Mais le Speculum futurorum temporum si diffusé dans les manuscrits n'eut aucun succès après l'avènement de l'imprimerie: seuls quelques extraits en furent publiés par le Bénédictin Jean-Baptiste Pitra dans son volume conçu pour apporter des compléments au volume 197 de la Patrologie latine intitulé de manière quelque peu trompeuse Sanctae Hildegardis abbatissae opera omnia. Les Nova sanctae Hildegardis opera publiées par Pitra en 1882 livrèrent entre autres au public deux séries de lettres intitulées «nova et altera series » et «novae epistolae », et firent accéder pour la première fois aux presses, bien que par quelques extraits seulement, le Speculum futurorum de Gebeno. On sait d'autant plus gré à José Carlos Santos Paz d'avoir livré au lecteur, dans son intégralité, non seulement un texte qui connut une telle fortune au Moyen Âge et qui restait inaccessible de nos jours, mais tout un riche dossier textuel : la lettre de Gebeno aux sœurs de Hildegarde, le petit traité De eisdem hereticis ex apocalypsi, un extrait du Liber divinorum operum et un autre de la lettre de Hildegarde au clergé de Trèves sur les signes avant-coureurs du schisme, sont ici proposés au lecteur dans une édition critique. En outre, José Carlos Santos Paz clôt son volume sur deux précieux appendices : la première version du chapitre 24 du Speculum, largement inspiré par une lettre de Hildegarde aux pasteurs de l'Église, et des extraits de la seconde rédaction du Speculum d'après un manuscrit de Munich (ms. München, BSB, clm 2619). Deux copieux indices, l'un des manuscrits et l'autre des noms d'auteurs et des œuvres anonymes, achèvent de faire de ce volume un très précieux outil de travail. 
INDEX

Index chronologique : Moyen Âge

Thèmes : Outils de travail, Sources

\section{AUTEUR}

LAURENCE MOULINIER-BROGI

Université Lumière-Lyon 2, UMR 5648 (CIHAM) 